\title{
Incretins and the specific mechanism of action of liraglutide, the first applicable human glucagon-like peptide I analog in the treatment of type 2 diabetes
}

\author{
This article was published in the following Dove Press journal: \\ Journal of Receptor, Ligand and Channel Research \\ 22 September 2010 \\ Number of times this article has been viewed
}

\section{Edoardo Mannucci \\ Caterina Lamanna}

Diabetes Agency, Careggi Teaching Hospital, Florence, Italy
Correspondence: Edoardo Mannucci Diabetologia Ponte Nuovo, Via delle Oblate, 4-50 I 4I Firenze, Italy

Tel +39055 7949742

Fax +39055 7949742

Email edoardo.mannucci@unifi.it
Abstract: Liraglutide is a once-daily glucagon-like peptide 1 (GLP-1) receptor agonist, approved for use as a treatment of type 2 diabetes. Like other drugs of the same class, liraglutide stimulates insulin secretion in a glucose-dependent fashion, has the potential of preventing $\beta$-cell mass decline, and inhibits food intake. In addition, experimental studies suggest that the GLP-1 receptor agonists could protect myocardium from ischemic injury, enhancing cardiac function. In clinical trials, liraglutide (in monotherapy or as add-on to 1 or 2 oral drugs) is as effective as, or more effective than, other agents (sulfonylureas, thiazolidinediones, dipeptidyl peptidase-4 inhibitors, insulin, and exenatide) in reducing hemoglobin $\mathrm{A}_{1 \mathrm{c}}$; induces weight loss; and has a blood pressure-lowering effect. The possible beneficial cardiovascular effects need to be confirmed by specifically designed long-term studies.

Keywords: liraglutide, type 2 diabetes, GLP-1 receptor agonist

\section{Incretins: introduction and effects on glucose metabolism}

Experiments performed over 30 years ago showed that oral glucose stimulates insulin secretion to a greater extent than a quantity of intravenous glucose determining the same glycemic increase. In order to justify this phenomenon, an oral glucose-induced secretion of gastrointestinal hormones capable of stimulating insulin secretion was postulated; those substances were indicated with the name of "incretins". ${ }^{1}$ The chemical nature of those substances remained obscure until the development of adequate laboratory methods allowed the identifications of several gut hormones, which are mainly secreted after meals and can modulate insulin secretion. Substances that can contribute to the incretin effect include secretin, glucagon, vasoactive intestinal peptide, glucose-dependent insulinotropic peptide (GIP), glucagon-like peptides 1 and 2 (GLP-1 and GLP-2), glicentin, and oxyntomodulin. Although all these substances modulate insulin secretion, only some have a relevant effect at physiological concentrations; in fact, about $80 \%$ of the incretin effect can be attributed to GLP-1 and, to a lesser extent, GIP. ${ }^{2}$

GLP-1 is mainly produced by endocrine cells dispersed in the mucosa of distal ileum, whereas GIP-producing cells are represented to a greater extent in jejunum., The secretion of both hormones is stimulated by carbohydrate-rich meals. The active form of GLP-1 binds to specific plasma membrane receptors, which have been 
demonstrated at the surface of several target cell types. ${ }^{2-4}$ The stimulation of GLP-1 receptors on pancreatic $\beta$ cell enhances glucose-induced insulin secretion; on the other hand, in whole islets (although not in isolated $\alpha$ cells), glucagon secretion is inhibited, at least in conditions of hypoglycemia. These 2 effects contribute to the reduction of postprandial hyperglycemia. ${ }^{4-7}$ The effects of GIP on $\alpha$ and $\beta$ cells are similar to that of GLP-1 although the latter hormone appears to be more relevant for the physiologic regulation of glucose metabolism in humans. ${ }^{7}$ Interestingly, prolonged exposure to GLP-1 appears to inhibit apoptosis and stimulate $\beta$-cell neogenesis, thus increasing $\beta$-cell mass. ${ }^{6,7}$ Although the effect of GLP-1 and GIP on glucose metabolism appears to be mainly due to the stimulation of insulin secretion, some experiments suggest that GLP-1 could also have some insulin-sensitizing effect, not entirely accounted for by the improvement of blood glucose and by the suppression of insulin secretion. ${ }^{8,9}$ Although this point remains controversial, ${ }^{10,11}$ both GLP-1 and GIP are rapidly inactivated by several peptidases, the most important of which is dipeptidyl peptidase-4 (DPP-4). ${ }^{7}$ Although the possibility of direct biological actions of the "inactivated" form of GLP-1, GLP-1(9-36) amide, has been suggested, this peptide is devoid of substantial hypoglycemic properties.

Postprandial circulating concentrations of active GLP-1 are reduced in type 2 diabetes; ${ }^{12-14}$ this is partly due to reduced secretion and enhanced degradation by DPP-4. Chronic hyperglycemia stimulated DPP-4 inhibition and activity, ${ }^{15,16}$ and it possibly blunts GLP-1 secretion, thus establishing a vicious cycle. Although a primary pathogenetic role of impaired GLP-1 response to meal is questionable, the disturbances of the incretin axis induced by elevated blood glucose are likely to contribute to the genesis of postprandial hyperglycemia in type 2 diabetes patients.

\section{Extraglycemic effects of incretins}

The actions of GLP-1 are summarized in Figure 1. The administration of active GLP-1, in rodents and in humans, enhances satiety and reduces food intake, promoting weight loss; ${ }^{3,4}$ however, treatment with GLP-1 receptor agonists is associated with reduction of body weight in type 2 diabetes patients. ${ }^{17,18}$ At supraphysiological concentrations

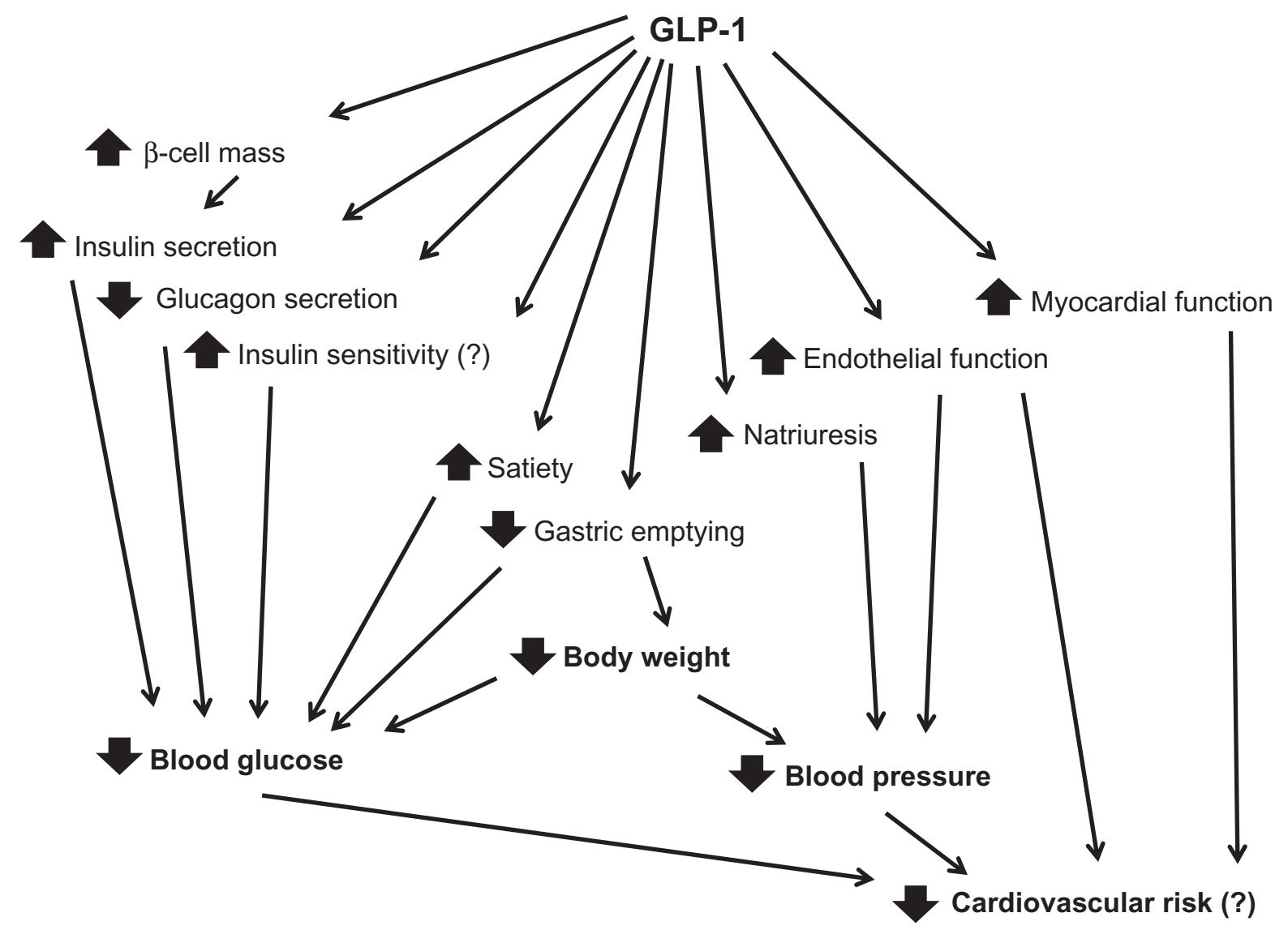

Figure I Biological actions of glucagon-like peptide I (GLP-I) in humans. 
of the hormone, active GLP-1 inhibits food intake by delaying gastric emptying. Even at lower concentrations, GLP-1 suppresses appetite through a direct effect on specific hypothalamic receptors; in humans, those central effects can be exerted by peripherally secreted GLP-1, which is able to cross the blood-brain barrier. ${ }^{2,19}$ Furthermore, GLP-1 is used as a neuromediator by a small population of neurons of the nucleus of the solitary tract, which participates to the regulation of appetite and satiety. ${ }^{20}$

Recent evidence shows that GLP-1 also has relevant direct effects on the cardiovascular system. ${ }^{2,21}$ In particular, the hormone enhances cardiac function, both in animal models and in humans, through a direct interaction with myocardiocytes. ${ }^{22,23}$ In rodents, the administration of active GLP-1 increases heart rate, possibly via a central action on the neuroregulation of heart rate, but no such effect has ever been observed in humans, either with native GLP-1 or GLP-1 receptor agonists. ${ }^{2}$ The cardiac effects of GLP-1 could be clinically relevant, as suggested by pilot studies showing that the short-term infusion of the hormone enhances myocardial function in patients with ischemic heart disease and/or heart failure. ${ }^{23,24}$

Experimental studies have shown that inactivated forms of GLP-1, such as GLP-1(9-36) amide, which is not an agonist of GLP-1 receptor, also have positive effects on myocardiocyte function. This suggests that the cardiac actions of GLP-1 could be partly mediated via a GLP-1 receptorindependent, and still obscure, pathway. ${ }^{7,25}$ Furthermore, it is possible that some cardiac effects of active GLP-1 are due to its inactivated metabolite GLP-1(9-36). For this reason, the myocardial actions of GLP-1 receptor agonists could be heterogeneous and different from those of native GLP-1.

Another important effect of GLP-1 is represented by its hypotensive action. Treatment of type 2 diabetes patients with GLP-1 receptor agonists, exenatide and liraglutide, produces a reduction in blood pressure, ${ }^{26,27}$ which is independent of weight loss and is attributable, at least partly, to a natriuretic effect of GLP-1 receptor stimulation. ${ }^{28}$

\section{Incretin-based therapies: DPP-4 inhibitors and GLP-I receptor agonists}

GLP-1 induces a glucose-dependent increase in insulin secretion and decrease in glucagon secretion; furthermore, it enhances $\beta$-cell function, inhibits $\beta$-cell apoptosis, induces a remarkable weight loss, and reduces blood pressure, while enhancing cardiac functions. All these properties make GLP-1 an ideal drug for the treatment of type 2 diabetes.
Unfortunately, its kinetics is incompatible with clinical use. In fact, the active form of the peptide, GLP-1(7-36) amide, is rapidly metabolized by several peptidases. The most important enzyme for GLP-1 (and GIP) degradation, DPP-4, cleaves a N-terminal dipeptide, producing the inactive GLP-1(9-36) amide, which is a weak GLP-1 receptor antagonist. ${ }^{3,7}$

To exploit the therapeutic potential of GLP-1, 2 different strategies have been explored. DPP-4 has been targeted for the development of inhibitors, which increase half-life and circulating concentrations of endogenous active GLP-1 and GIP. ${ }^{29,30}$ The class of DPP-4 inhibitors includes several molecules (such as vildagliptin, sitagliptin, saxagliptin, alogliptin, linagliptin), all administered orally once or twice daily. DPP-4 inhibitors have been shown to reduce plasma glucose and $\mathrm{HbA}_{1 \mathrm{c}}$ to a somewhat lower extent than other available drugs if used in monotherapy. ${ }^{31}$ They can be combined with insulin-sensitizing drugs (metformin and thiazolidinediones) and/or insulinotropic agents (sulfonylureas). DPP-4 inhibitors do not induce weight gain, nor weight loss, and, unless they are associated with stimulators of insulin secretion, they do not induce any relevant hypoglycemic risk. ${ }^{17,31}$ In general, their tolerability and safety profile seem to be extremely favorable; in fact, mild upper respiratory tract or urinary tract infections are the only adverse events registered, although long-term, large-scale studies are still lacking.

An alternative approach is represented by the development of GLP-1 receptor agonists, which are resistant to the action of DPP-4, so that their kinetics is compatible with clinical use. Unlike DPP-4 inhibitors, GLP-1 receptor agonists need to be administered through subcutaneous injections. The first molecule of this class, exenatide, has a 1.5-hour half-life, which warrants plasma concentrations within the therapeutic range for 4-5 hours after administration. Used twice daily, before main meals, exenatide induces a relevant reduction of plasma glucose and $\mathrm{HbA}_{1 \mathrm{c}}$ in comparison with placebo, associated with remarkable weight loss. ${ }^{17,18}$ Longer-term data from uncontrolled extensions of randomized clinical trials show that exenatide has a sustained hypoglycemic and weight-reducing effect for as long as 30 months. ${ }^{26}$ Unless associated with a sulfonylurea, this drug does not induce hypoglycemia. The main adverse event is represented by nausea and vomiting, which occurs in about one-third of all patients; these side effects are usually transient, so that it leads to discontinuation of therapy in only a minority of patients. ${ }^{18}$ The only severe adverse event that has been associated with exenatide treatment to date is represented by rare cases of usually mild acute pancreatitis. Exenatide 
is indicated as add-on treatment in patients inadequately controlled with metformin and/or sulfonylureas. Some guidelines recommend its use as a second-line therapy in metformin monotherapy failure, ${ }^{32}$ whereas others suggest to prescribe exenatide, as an alternative to insulin, in patients failing to the combination of metformin and sulfonylureas. ${ }^{33}$ In fact, although most trials were performed in subjects inadequately controlled with 2 drugs, physiopathological considerations suggest that GLP-1 receptor agonists should be more useful in earlier stages of the disease.

DPP-4 inhibitors and GLP-1 receptor agonists, although they act on the same pathway, have a quite different clinical profile (Table 1). Apart from the obvious difference in the route of administration (oral for DPP-4 inhibitors and subcutaneous for GLP-1 receptor agonists), DPP-4 inhibitors have a superior tolerability profile with very few patients complaining of adverse events, whereas GLP-1 receptor agonists are associated with nausea. Conversely, GLP-1 receptor agonists unlike DPP-4 inhibitors induce weight loss. Furthermore, direct comparisons show a greater efficacy of exenatide and liraglutide with respect to the DPP-4 inhibitor sitagliptin. ${ }^{33,34}$ These differences are due to the fact that treatment with DPP-4 inhibitors induces a 3- to 4-fold increase of active GLP-1 concentrations, whereas circulating concentrations of GLP-1 receptor agonists (expressed as molarity) can reach levels as high as 15 times those of endogenous GLP-1. ${ }^{33}$

\section{Liraglutide: mechanisms of action, preclinical studies, and early clinical development}

Liraglutide was developed as a long-acting, DPP-4 resistant, GLP-1 receptor agonists based on the structure of native GLP-1, which was modified by replacing a lysine with an arginine residue at position 34 and by attaching a C16 acyl chain via a glutamoyl spacer to lysine at position $26 .{ }^{27}$ This structural modification allowed the development of a GLP-1like molecule with an affinity for the GLP-1 receptor similar to

Table I Comparison of GLP-I receptor agonists and DPP-4 inhibitors

\begin{tabular}{lll}
\hline Characteristics & $\begin{array}{l}\text { GLP-I receptor } \\
\text { agonists }\end{array}$ & DPP-4 inhibitors \\
\hline Route of administration & Subcutaneous & Oral \\
Side effects & Nausea, vomiting & Rare $^{\mathrm{a}}$ \\
Effect on $\mathrm{HbA}_{\text {Ic }}$ & Marked reduction & Moderate reduction \\
Effect on body weight & Reduction & Neutral \\
Effect on blood pressure & Mild reduction & $?$ \\
\hline
\end{tabular}

Notes: ${ }^{a} \mathrm{An}$ increased incidence of nasopharyngitis has been reported. Abbreviations: GLP-I, glucagon-like peptide I; DPP-4, dipeptidyl peptidase-4. that of native GLP-1 but with a longer half-life. ${ }^{27}$ Liraglutide, which has a half-life of about 13 hours, can be administered once daily (at the dose of $1.2-1.8 \mathrm{mg}$ ), reaching and maintaining therapeutic concentrations throughout 24 hours. ${ }^{35}$ In fact, liraglutide is relatively resistant to DPP-4 activity; furthermore, the fatty acid chain promotes binding to albumin, which reduced the renal clearance of the molecule. ${ }^{36}$

Liraglutide has a direct effect on pancreatic $\beta$ cells, stimulating insulin biosynthesis and secretion. ${ }^{27}$ The administration of liraglutide in several animal models and in human stimulates glucose-dependent insulin release, while enhancing the inhibitory effect of hyperglycemia on glucagon secretion. This leads to a dose-dependent reduction of plasma glucose. At the same time, liraglutide suppresses food intake and induces weight loss in several animal models. ${ }^{27,35}$ Available evidence shows that in vivo chronic administration of liraglutide increases $\beta$-cell mass in several rodent models of diabetes; furthermore, the drug inhibits $\beta$-cell apoptosis in human islets in vitro. ${ }^{27}$

Phase 2 clinical trials showed a dose-dependent effect of liraglutide on blood glucose and $\mathrm{HbA}_{1 \mathrm{c}}$ in the range of $0.5-2.0 \mathrm{mg} / \mathrm{d}$, allowing the identification of $0.6,1.2$, and $1.8 \mathrm{mg} / \mathrm{d}$ as the most convenient dosage for the treatment of type 2 diabetes. ${ }^{27,35}$ The increase of daily doses over $2 \mathrm{mg} / \mathrm{d}$ does not produce additional effects on glucose, but it induces a further weight gain. The maximum weight-reducing efficacy is observed at about $3 \mathrm{mg} / \mathrm{d} .{ }^{36}$ For this reason, although the maximum recommended dose for diabetes is $1.8 \mathrm{mg} / \mathrm{d}$, if liraglutide was developed for the treatment of obesity, the dose range for that indication could be substantially higher. Interestingly, the effect of liraglutide on body weight in patients with obesity is greater than that of a drug specifically indicated for obesity, orlistat, even at the doses used for the treatment of diabetes $(1.2-1.8 \mathrm{mg} / \mathrm{d}) .{ }^{36}$

\section{Liraglutide: clinical profile}

Most available information on liraglutide is derived from a wide program of phase 3 trials, the Liraglutide Effect and Action in Diabetes (LEAD) studies, and the characteristics of which are summarized in Table 2. These trials explore the effects of liraglutide in monotherapy and in combination with 1 or 2 oral agents; in most instances, the effect of liraglutide is compared with both placebo and an active drug.

When used as monotherapy (which is not among the approved indications of the drug), liraglutide reduces $\mathrm{HbA}_{1 \mathrm{c}}$ to a greater extent than a maximal dose of the sulfonylurea glimepiride with a much lower hypoglycemic risk and no weight gain; the only disadvantage of liraglutide is a 
Table 2 Main clinical trials with liraglutide

\begin{tabular}{llll}
\hline Trial & Condition & Add-on to & Comparator \\
\hline LEAD-3 $^{37}$ & T2DM & None & Glimepiride \\
LEAD-2 $^{38}$ & T2DM & Metformin & Placebo, glimepiride \\
LEAD-1 $^{39}$ & T2DM & Sulfonylurea & Placebo, rosiglitazone \\
LEAD-4 $^{40}$ & T2DM & Metformin + TZD & Placebo \\
LEAD-5 $^{41}$ & T2DM & Metformin + SU & Placebo, glargine \\
LEAD-6 $^{42}$ & T2DM & Metformin \pm SU & Exenatide \\
Pratley $^{34}$ & T2DM & Metformin & Sitagliptin \\
Astrup $^{36}$ & Obesity & None & Placebo, orlistat
\end{tabular}

Abbreviations: LEAD, liraglutide effect and action in diabetes; T2DM, type 2 diabetes mellitus; TZD, thiazolidinediones; SU, sulfonylureas.

higher incidence of nausea. ${ }^{37}$ Used as add-on to metformin, liraglutide induces a dose-dependent decrease of $\mathrm{HbA}_{1 \mathrm{c}}$ with respect to placebo, similar to that obtained with glimepiride; however, in comparison with sulfonylurea, liraglutide has the advantage of inducing weight loss instead of weight gain. Furthermore, unlike glimepiride, the addition of liraglutide to metformin is not associated with hypoglycemia. ${ }^{38}$ In metformin-intolerant patients inadequately controlled with sulfonylureas, the addition of liraglutide is more effective on $\mathrm{HbA}_{1 \mathrm{c}}$, at least in the short-term, than thiazolidinedione; 39 none of the 2 drugs induces hypoglycemia, whereas their effects on body weight are divergent (weight loss with liraglutide and weight gain with thiazolidinediones).

Some trials of the LEAD program have been designed to explore the effect of the drug as add-on therapy in patients inadequately controlled with 2 oral drugs. When added to a combination of metformin and thiazolidinediones, liraglutide produces a further reduction of $\mathrm{HbA}_{1 \mathrm{c}}{ }^{40}$ One of the trials of the LEAD program compared liraglutide with a basal insulin (glargine), both administered once daily, in patients who had an elevated $\mathrm{HbA}_{1 \mathrm{c}}$ despite treatment with a combination of metformin and sulfonylureas. In this study, a similar effect on metabolic control was observed with the 2 treatments, with a divergent action on body weight (increased by insulin and decreased by liraglutide). ${ }^{41}$

Some further trials, which were not part of the original phase 3 program, have compared liraglutide with other incretinbased therapies. In particular, the LEAD-6 trial was designed to compare liraglutuide with exenatide. After 26 weeks of treatment, liraglutide produced a greater reduction of $\mathrm{HbA}_{1 \mathrm{c}}$ than exenatide; in fact, the newer GLP-1 receptor agonists reduced fasting glucose to a greater extent, whereas its overall effect on postprandial hyperglycemia was similar to that of exenatide. ${ }^{42}$ This difference in efficacy is consistent with the results of placebo-controlled trials, in which liraglutide, on average, produces a greater reduction of $\mathrm{HbA}_{1 \mathrm{c}}$ than exenatide. ${ }^{18}$ The difference between the 2 drugs can be explained on the basis of their kinetic profile. In fact, exenatide, which has a rather short half-life, when administered twice daily maintains circulating concentrations within the therapeutic range only for 10-12 hours per day, whereas liraglutide action is extended over 24 hours per day, producing a greater effect on plasma glucose. The reduced variability of plasma drug levels produces another favorable consequence, ie, a more rapid induction to tolerance with respect to nausea. In fact, although the proportion of patients experiencing nausea at any time during the trial is similar to either of the 2 drugs, this side effect appears to be more transient, and therefore better tolerated, with liraglutide than with exenatide. Furthermore, liraglutide, which has a greater homology to human GLP-1, is less immunogenic than exenatide, so that its administration is associated with production of specific antibodies only in a minority of cases. However, the clinical relevance of this latter phenomenon is uncertain; in fact, the possibility that antiexenatide antibodies may blunt the long-term response to treatment is controversial. On the other hand, the effects of liraglutide on body weight and blood pressure are similar to those of exenatide. ${ }^{42}$

Another trial compared liraglutide with the DPP-4 inhibitor sitagliptin..$^{34}$ Added to metformin, the GLP-1 receptor agonist showed a greater efficacy on $\mathrm{HbA}_{1 \mathrm{c}}$ and fasting glucose after 26 weeks, associated with a significant weight loss; conversely, sitagliptin had a higher tolerability, whereas liraglutide induced gastrointestinal symptoms in a fraction of cases. This result is consistent with that of a previous, shorter-term trial comparing exenatide with sitagliptin, ${ }^{33}$ showing that GLP-1 receptor agonists have a greater efficacy than DPP-4 inhibitors.

Overall, the safety profile of liraglutide appears to be satisfactory. The main side effects, nausea and vomiting, are usually transient; no major adverse events emerge from phase 3 clinical trials. ${ }^{18}$ There are still no available data about the possible risk of pancreatitis, which has been associated with GLP-1 receptor agonist therapy; however, the drug should 
not be used in patients with previous pancreatitis or with cholelitiasis. In rodent models, treatment with high-dose liraglutide induces hyperplasia of thyroid C-cells and, in some cases, thyroid medullary carcinoma; however, no cases of medullary carcinoma have been observed to date with liraglutide, and no relevant increase of circulating calcitonin has been detected during phase 3 clinical trials. It has been observed that, in comparison with humans, rodents have a much higher incidence of medullary carcinoma and a greater density of C-cells in the thyroid gland. Furthermore, the expression of GLP-1 receptors is by far higher in rodent than in human thyroid C-cells. However, longer-term observations on larger numbers of patients are needed to exclude the possibility of an increased risk of medullary carcinoma associated with liraglutide or other incretin-based therapies. ${ }^{43,44}$

Liraglutide has an interesting profile of action on cardiovascular risk factors, reducing, at the same time, blood glucose, body weight, and blood pressure; this drug also improves lipid profile, possibly through the reduction of weight. ${ }^{2,27}$ It is also possible that some of the beneficial effects of GLP-1 on myocardium described above are shared by GLP-1 receptor agonists, although this point still needs to be clarified. There is a certain expectation that long-term treatment with liraglutide, or other drugs of the same class, could be associated with a reduction of cardiovascular risk; however, the issue can be resolved only through appropriately sized randomized trials of appropriate duration. In available studies, there is no detectable increase of incidence of major cardiovascular events in patients treated with GLP-1 receptor agonists, ${ }^{18}$ but the size of the samples enrolled and the duration of observation are largely insufficient to draw any conclusion. The US Food and Drug Administration requires, for newly approved drugs for type 2 diabetes, that such randomized trials should be performed as to exclude any increase of cardiovascular risk, with an upper limit of confidence interval for major cardiovascular events below 1.30. When this information will be available for liraglutide, we will have a clearer picture of the long-term effects of treatment with GLP-1 receptor agonists.

An overall consideration of available evidence on efficacy and safety of this drug led the US Food and Drug Administration to approve the use of liraglutide in type 2 diabetes patients failing to 1 or 2 oral agents. ${ }^{43}$

\section{How to use liraglutide in clinical practice}

Type 2 diabetes is a complex disease, which requires more than one drug in most cases. In order to attain recommended glycemic goals, most patients receive a complex treatment with different combinations of insulin sensitizers and insulinotropic agents. Although most specialists agree that the first-choice drug, unless contraindicated, is metformin, there is no consensus on what should be preferred as add-on treatment when metformin monotherapy is not sufficient. A variety of drugs, including thiazolidinediones, sulfonylureas, glinides, $\alpha$-glucosidase inhibitors, DPP-4 inhibitors, and insulin, can be added to metformin if necessary; in some cases, combinations of 3 of these agents can be prescribed.

Current guidelines are discordant on the place of GLP-1 receptor agonists in the algorithm for pharmacological treatment of type 2 diabetes. The American Diabetes Association/European Association for Study of Diabetes consensus on treatment of type 2 diabetes ${ }^{32}$ considers GLP-1 receptor agonists a possible option in patients inadequately controlled by metformin monotherapy. Considering that the main mechanism of hypoglycemic action of GLP-1 receptor agonists is the stimulation of glucose-dependent insulin secretion, it seems logical to suppose that these drugs could be more effective in early stages of diabetes, when $\beta$-cell mass and function are still relatively preserved. Other documents, such as the National Institute for Health and Clinical Excellence guidelines, ${ }^{33}$ recommend GLP-1 receptor agonists only as add-on to sulfonylureas and metformin in patients with obesity. This position is based on the fact that most trials (and all the available trials vs active comparators) with exenatide have been performed in patients failing to combinations of sulfonylureas and metformin. The clinical program for liraglutide described above is much wider and includes a number of trials showing that this drug has at least the same efficacy as, or a greater efficacy than, sulfonylureas and thiazolidinediones also in earlier stages of the disease (ie, in monotherapy or in combination with either metformin or sulfonylureas). Therefore, the available data justify the use of liraglutide in several different steps of therapy for diabetes.

Monotherapy, although of potential interest, is not among the currently approved indications. The only one monotherapy trial, showing superiority in comparison with glimepiride, ${ }^{37}$ was not considered sufficient by health authorities to grant this indication. In fact, there is no evidence of superiority of liraglutide over metformin, which is universally considered the drug of choice as a first-line treatment of type 2 diabetes.

In patients inadequately controlled by metformin monotherapy, liraglutide can be used as add-on treatment. In these subjects, there are a variety of therapeutic options, 
and the most commonly used is the addition of sulfonylureas or thiazolidinediones. In comparison with sulfonylureas, liraglutide has at least the same efficacy on $\mathrm{HbA}_{1 \mathrm{c}}$ with no hypoglycemic risk; on the other hand, it induces weight loss instead of weight gain. ${ }^{38}$ Furthermore, liraglutide has a greater efficacy than DPP-4 inhibitors. ${ }^{34}$ No direct comparisons with thiazolidinediones in combination with metformin are available to date, and the only published trial vs rosiglitazone is performed in combination with sulfonylureas, ${ }^{39}$ however, in that trial, liraglutide had a greater efficacy than rosiglitazone with divergent effects on body weight. On the other hand, the route of administration could represent an obstacle for treatment with GLP-1 receptor agonists, particularly in patient who are not motivated to lose weight. Despite this limitation, liraglutide treatment should always be considered in patients with obesity inadequately controlled on metfomin monotherapy.

Type 2 diabetes patients who are intolerant to metformin or who show contraindications to metformin are usually treated with sulfonylureas or thiazolidinediones. If sulfonylurea monotherapy is insufficient, the addition of liraglutide improves metabolic control to a greater extent than a thiazolidinedione, while reducing body weight. ${ }^{39}$

In patients who do not reach (or maintain) glycemic goals despite treatment with 2 oral drugs (metformin plus sulfonylureas, or metformin and thiazolidinediones), liraglutide is an interesting alternative to insulin. ${ }^{41}$ In fact, it has the advantage of a low hypoglycemic risk (if used in combination with metformin and thiazolidinediones $)^{40}$ with a lower need for intensified blood glucose self-monitoring; furthermore, it has a weight-reducing effect, and it does not induce fluid retention. ${ }^{40,41}$ At the same time, we should be aware of the fact that therapeutic failures could be more frequent when GLP-1 receptor agonists are used in patients with very little residual $\beta$-cell mass and function.

\section{Conclusion}

GLP-1 receptor agonists represent a very interesting new option for the treatment of type 2 diabetes. They offer the opportunity of reducing blood glucose and $\mathrm{HbA}_{1 \mathrm{c}}$ with a low hypoglycemic risk, associated with weight loss. In comparison with DPP-4 inhibitors, GLP-1 receptor agonists have the advantage of a greater efficacy and a remarkable weight reducing effect. The potential protection from decline of $\beta$-cell mass and function is an interesting perspective, which needs to be confirmed by longer-term studies. Similarly, the profile of action on cardiovascular risk seems to be favorable, but specifically designed trials on major cardiovascular events are required to verify the effect of GLP-1 receptor agonists in long-term treatment. Within this class, liraglutide is the first agent with a sufficiently favorable kinetics to allow once-daily administration; furthermore, the possibility of maintaining circulating drug concentrations within the therapeutic range throughout the day accounts for a greater efficacy of liraglutide in comparison with earlier agents of the same class.

\section{Disclosure}

Edoardo Mannucci has received consultancy fees and reserach grants from Novo Nordisk.

\section{References}

1. Nauck M, Stöckmann F, Ebert R, Creutzfeldt W. Reduced incretin effect in type 2 (non-insulin-dependent) diabetes. Diabetologia. 1986; 29(1):46-52.

2. Mannucci E, Rotella CM. Future perspectives on glucagon-like peptide-1, diabetes and cardiovascular risk. Nutr Metab Cardiovasc Dis. 2008;18(9):639-645.

3. Baggio LL, Drucker DJ. Biology of incretins: GLP-1 and GIP. Gastroenterology. 2007;132(6):2131-2157.

4. Rotella CM, Pala L, Mannucci E. Glucagon-like peptide 1 (GLP-1) and metabolic diseases. $J$ Endocrinol Invest. 2005;28(8):746-758.

5. Mudaliar S, Henry RR. Effects of incretin hormones on beta-cell mass and function, body weight, and hepatic and myocardial function. Am J Med. 2010;123(Suppl 3):S19-S27.

6. Karaca M, Magnan C, Kargar C. Functional pancreatic beta-cell mass: involvement in type 2 diabetes and therapeutic intervention. Diabetes Metab. 2009;35(2):77-84. Epub 2009 Feb 28.

7. Holst JJ. The physiology of glucagon-like peptide-1. Physiol Rev. 2007; 87(4):1409-1439.

8. Gutniak M, Orskov C, Holst JJ, Ahren B, Efendic S. Antidiabetogenic effects of glucagon-like peptide-1 (7-36) amide in normal subjects and patients with diabetes mellitus. $N$ Engl J Med. 1992;326(20): $1316-1322$.

9. Sandhu H, Wiesenthal SR, McDonald PE, et al. Glucagon-like peptide-1 increases insulin sensitivity in depancreatized dogs. Diabetes. 1999;48(5):1045-1053.

10. Orskov L, Holst JJ, Moller J, et al. GLP-1 does not acutely affect insulin sensitivity in healthy man. Diabetologia. 1996;39(10):1227-1232.

11. Toft-Nielson M, Madsbad S, Holst JJ. The effect of glucagon-like peptide I (GLP-1) on glucose elimination in healthy subjects depends on the pancreatic glucoregulatory hormones. Diabetes. 1996;45(5): 552-556.

12. Mannucci E, Ognibene A, Cremasco F, et al. Glucagon-like peptide (GLP)-1 and leptin concentrations in obese patients with type 2 diabetes mellitus. Diabet Med. 2000;17(10):713-719.

13. Vilsbøll T, Krarup T, Deacon CF, Madsbad S, Holst JJ. Reduced postprandial concentrations of intact biologically active glucagon-like peptide 1 in type 2 diabetic patients. Diabetes. 2001;50(3):609-613.

14. Vilsbøll T, Krarup T, Sonne J, et al. Incretin secretion in relation to meal size and body weight in healthy subjects and people with type 1 and type 2 diabetes mellitus. J Clin Endocrinol Metab. 2003;88(6):2706-2713.

15. Pala L, Mannucci E, Pezzatini A, et al. Dipeptidyl peptidase-IV expression and activity in human glomerular endothelial cells. Biochem Biophys Res Commun. 2003;310(1):28-31.

16. Mannucci E, Pala L, Ciani S, et al. Hyperglycaemia increases dipeptidyl peptidase IV activity in diabetes mellitus. Diabetologia. 2005;48(6):1168-1172.

17. Amori RE, Lau J, Pittas AG. Efficacy and safety of incretin therapy in type 2 diabetes: systematic review and meta-analysis. JAMA. 2007;298(2):194-206. 
18. Monami M, Marchionni N, Mannucci E. Glucagon-like peptide-1 receptor agonists in type 2 diabetes: a meta-analysis of randomized clinical trials. Eur J Endocrinol. 2009;160(6):909-917.

19. Orskov C, Poulsen SS, Moller M, Holst JJ. Glucagon-like peptide I receptors in the subfornical organ and the area postrema are accessible to circulating glucagon-like peptide I. Diabetes. 1996;45(6):832-835.

20. Drucker DJ, Asa S. Glucagon gene expression in vertebrate brain. J Biol Chem. 1988;263(27):13475-13478.

21. Giorgino F, Natalicchio A, Leonardini A, Laviola L. Exploiting the pleiotropic actions of GLP-1 for the management of type 2 diabetes mellitus and its complications. Diabetes Res Clin Pract. 2007;78(3): S59-S67.

22. Nikolaidis LA, Elahi D, Hentosz T, et al. Recombinant glucagon-like peptide-1 increases myocardial glucose uptake and improves left ventricular performance in conscious dogs with pacing-induced dilated cardiomyopathy. Circulation. 2004;110(8):955-961.

23. Sokos GG, Nikolaidis LA, Mankad S, Elahi D, Shannon RP. Glucagon-like peptide-1 infusion improves left ventricular ejection fraction and functional status in patients with chronic heart failure. J Card Fail. 2006;12(9):694-699.

24. Nikolaidis LA, Mankad S, Sokos GG, et al. Effects of glucagon-like peptide-1 in patients with acute myocardial infarction and left ventricular dysfunction after successful reperfusion. Circulation. 2004; 109(8):962-965

25. Ban K, Kim KH, Cho CK, et al. Glucagon-like peptide (GLP)-1(9-36) amide-mediated cytoprotection is blocked by exendin(9-39) yet does not require the known GLP-1 receptor. Endocrinology. 2010;151(4): $1520-1531$.

26. Klonoff DC, Buse JB, Nielsen LL, et al. Exenatide effects on diabetes, obesity, cardiovascular risk factors and hepatic biomarkers in patients with type 2 diabetes treated for at least 3 years. Curr Med Res Opin. 2008;24(1):275-286.

27. Vilsbøll T. Liraglutide: a once-daily GLP-1 analogue for the treatment of type 2 diabetes mellitus. Expert Opin Investig Drugs. 2007;16(2): 231-237.

28. Carraro-Lacroix LR, Malnic G, Girardi AC. Regulation of $\mathrm{Na}+\mathrm{H}+$ exchanger NHE3 by glucagon-like peptide 1 receptor agonist exendin-4 in renal proximal tubule cells. Am J Physiol Renal Physiol. 2009;297(6):F1647-F1655.

29. Drucker DJ, Nauck MA. The incretin system: glucagon-like peptide-1 receptor agonists and dipeptidyl peptidase- 4 inhibitors in type 2 diabetes. Lancet. 2006;368(9548):1696-1705.

30. Inzucchi SE, McGuire DK. New drugs for the treatment of diabetes; Part II: incretin-based therapy and beyond. Circulation. 2008;117(4): 574-584.

31. Monami M, Iacomelli I, Marchionni N, Mannucci E. Dipeptidylpeptidase 4 inhibitors in type 2 diabetes: a meta-analysis of randomized clinical trials. Nutr Metab Cardiovasc Dis. 2010;20(4):224-235.

32. Nathan DM, Buse JB, Davidson MB, et al; American Diabetes Association; European Association for Study of Diabetes. Medical management of hyperglycemia in type 2 diabetes: a consensus algorithm for the initiation and adjustment of therapy: a consensus statement of the American Diabetes Association and the European Association for the Study of Diabetes. Diabetes Care. 2009;32(1):193-203.
33. Type 2 diabetes: the management of type 2 diabetes. Available from: http://www.nice.org.uk/CG66. Accessed May 28, 2008.

33. DeFronzo RA, Okerson T, Viswanathan P, Guan X, Holcombe JH, MacConell L. Effects of exenatide versus sitagliptin on postprandial glucose, insulin and glucagon secretion, gastric emptying, and caloric intake: a randomized, cross-over study. Curr Med Res Opin. 2008; 24(10):2943-2952.

34. Pratley RE, Nauck M, Bailey T, et al; 1860-LIRA-DPP-4 Study Group. Liraglutide versus sitagliptin for patients with type 2 diabetes who did not have adequate glycaemic control with metformin: a 26-week, randomised, parallel-group, open-label trial. Lancet. 2010;375: 1447-1456.

35. Russell-Jones D. Molecular, pharmacological and clinical aspects of liraglutide, a once-daily human GLP-1 analogue. Mol Cell Endocrinol. 2009;297(1-2):137-140.

36. Astrup A, Rössner S, van Gaal L, et al; NN8022-1807 Study Group. Effects of liraglutide in the treatment of obesity: a randomised, doubleblind, placebo-controlled study. Lancet. 2009;374(9701):1606-1616.

37. Garber A, Henry R, Ratner R, et al; LEAD-3 (Mono) Study Group. Liraglutide versus glimepiride monotherapy for type 2 diabetes (LEAD-3 Mono): a randomised, 52-week, phase III, double-blind, parallel-treatment trial. Lancet. 2009;373(9662):473-481.

38. Nauck M, Frid A, Hermansen K, et al; LEAD-2 Study Group. Efficacy and safety comparison of liraglutide, glimepiride, and placebo, all in combination with metformin, in type 2 diabetes: the LEAD (liraglutide effect and action in diabetes)-2 study. Diabetes Care. 2009; 32(1):84-90.

39. Marre M, Shaw J, Brändle M, et al; LEAD-1 SU Study Group. Liraglutide, a once-daily human GLP-1 analogue, added to a sulphonylurea over 26 weeks produces greater improvements in glycaemic and weight control compared with adding rosiglitazone or placebo in subjects with type 2 diabetes (LEAD-1 SU). Diabet Med. 2009;26(3):268-278

40. Zinman B, Gerich J, Buse JB, et al; LEAD-4 Study Investigators. Efficacy and safety of the human glucagon-like peptide-1 analog liraglutide in combination with metformin and thiazolidinedione in patients with type 2 diabetes (LEAD-4 Met+TZD). Diabetes Care. 2009;32(7):1224-1230.

41. Russell-Jones D, Vaag A, Schmitz O, et al; Liraglutide Effect and Action in Diabetes 5 (LEAD-5) met+SU Study Group. Liraglutide vs insulin glargine and placebo in combination with metformin and sulfonylurea therapy in type 2 diabetes mellitus (LEAD-5 met+SU): a randomised controlled trial. Diabetologia. 2009;52(10):2046-2055.

42. Buse JB, Rosenstock J, Sesti G, et al; LEAD-6 Study Group. Liraglutide once a day versus exenatide twice a day for type 2 diabetes: a 26-week randomised, parallel-group, multinational, open-label trial (LEAD-6). Lancet. 2009;374(9683):39-47.

43. Parks M, Rosebraugh $\mathrm{C}$. Weighing risks and benefits of liraglutide-The FDA's review of a new antidiabetic therapy. $N$ Engl J Med. 2010;362: 774-777.

44. Bjerre Knudsen L, Madsen LW, Andersen S, et al. Glucagon-like peptide-1 receptor agonists activate rodent thyroid $\mathrm{C}$-cells causing calcitonin release and C-cell proliferation. Endocrinology. 2010;151(4): 1473-1486.
Journal of Receptor, Ligand and Channel Research

\section{Publish your work in this journal}

The Journal of Receptor, Ligand and Channel Research is an international, peer-reviewed, open access, online journal. The journal welcomes laboratory and clinical findings in the fields of biological receptors, ligands, channel and signal transduction research including: receptors and signalling; ligands; transporters, pores and channels; binding and activation; receptor

\section{Dovepress}

regulation; role of receptors in diseases and their treatment; molecular basis of membrane structure and functions; molecular models of membranes. The manuscript management system is completely online and includes a very quick and fair peer-review system. Visit http://www.dovepress.com/ testimonials.php to read real quotes from published authors. 